\title{
Ability of automatic detection of conflict between planes in flight simulations with the help of expert system
}

\author{
N. Bartošová \\ Department of Air Defence Systems, University of Defence \\ Brno, Czech Republic \\ bn1111@seznam.cz
}

\begin{abstract}
This article examines options for applying expert systems for the needs of identification of conflict situations between planes in flight simulations, which are applied during basic training of air traffic controllers. It focuses on the conditions for basic training of military air traffic controllers and presents the use of rule systems to automatic detection of conflict between planes within a basic training polygon. The system of rules is a part of the expert system, consisting of realisation of tasks for identifying optimum resolution of conflict situations in selected types of simulations.
\end{abstract}

Keywords-conflict situations between planes, expert system, simulation of air situations, rule systems.

\section{INTRODUCTION}

The issue of application of artificial intelligence for the needs of identifying conflict situations (reduction of the minimum separation limits between planes) has already been addressed in articles [1], [2], which resolved a proposed polygon for performing basic training and evaluated approaches to the task of finding optimum resolution of conflicts between planes with the help of a knowledge-based approach. This article will focus on the first of three highlighted tasks, which have been set for undertaking of the task of finding an optimum resolution of conflict, specifically the task of automatic identification of conflict situations between planes in selected basic training simulations.

\section{REALISATION OF TRAINING IN CZECH ARMY'S CONDITIONS}

Military training centres are currently undergoing extensive reorganisation. Although in the future they are seriously considering purchasing training in the civil sphere, it can be expected that military air traffic controllers who provide services at Control and Reporting Centre (CRC) will continue to be trained within the Czech Army's environment. For these purposes, it would be necessary to expand the current form of training to include a new and for military centres a yet unresolved examination of basic training [1], [2].

In accordance with the philosophy of basic training, a separate set of trivial flight situations should be created, which would be designed to enable adoption of basic (procedural) approaches in a simple polygon proposed for this purpose. Basic training stems from the need to teach students how to apply the general principles for air traffic control, regardless of the environment and type of provided service. Therefore, it should represent the student's first contact with the practical side of air traffic control, during which the student should adopt simple means of resolving conflicts (word with the speeds of individual planes, procedural resolution of conflicts, etc.) and thus examine the issue of behaviour of individual planes within tactical-technical data (manoeuvrability, travelling speed, etc.).

\section{IDENTIFICATION OF CONFLICTS BETWEEN PLANES}

Generally said, conflict situations between planes are determined by finding a crisis interval of simulation - Ik (the interval of the horizontal limit reduction) and by pinpointing crisis flight levels of simulation - Fk (the set of conflict flight levels achieved by planes within the crisis interval of simulation). In the basic training, the value of minimum separation limits could be over - sized according to relative position of the tracks.

In the real environment, planes can encounter each other at the same, opposite and crossing routes [3]. In the environment proposed for the needs of basic training, planes move only in the direction of two proposed angles, and therefore they have routes along which planes fly as follows:

- the same routes form an angle of zero,

- routes opposite each other form an angle of 180 degrees,

- crossing tracks form an angle of 90 degrees or 270 degrees.

Resolution of the task of automatic identification of conflict - reduced minimum of the separation limits between two planes - was specified for a situation when planes encounter each other in crossing routes. An example of a simple simulation, in which planes encounter each other on crossing routes in the environment of a polygon for basic training, is shown in Figure 1. 


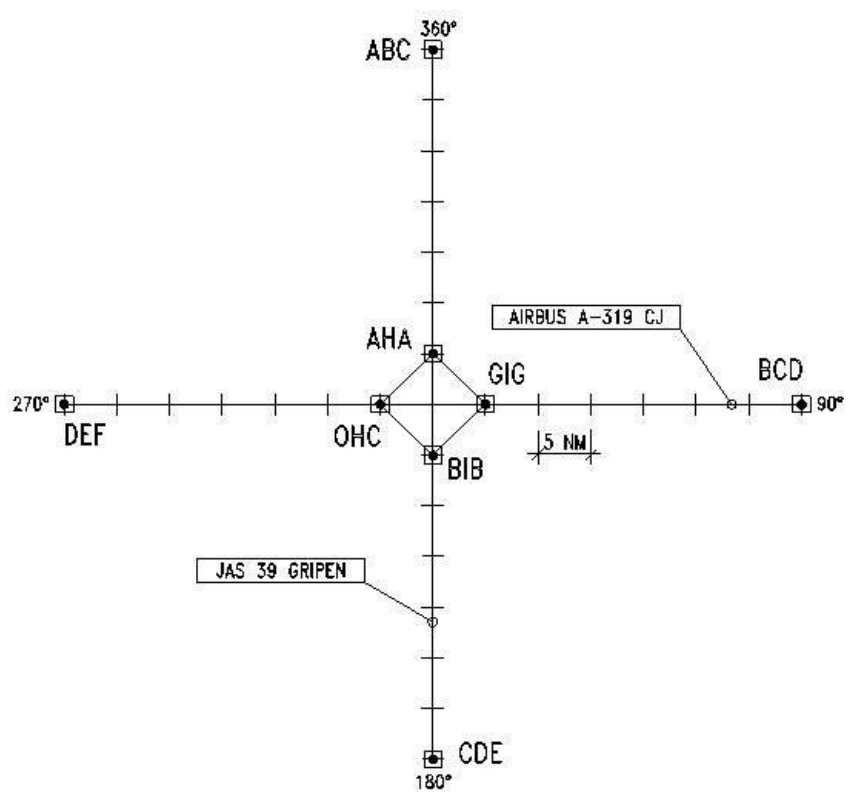

Figure 1 Planes on crossing routes

Unlike regularly used environments for training (the real environment of the Czech Republic's air space), a simple rosette is considered, consisting of two axes diagonal to each other, along with planes fly. On the horizontal axis, we see an Airbus A-319 shortly after entering the polygon (via the BCD beacon), flying towards the GIG and OHC beacons, which form entry and exit points to and from the conflict zones, where an effort to create conflict situations will be focused on when planning training. The plane leaves the polygon passing the DEF beacon. A JAS-39 Gripen plane (hereinafter referred to as "plane A") is captured in the picture in a vertical angle shortly after entering the polygon (via the CDE beacon), and it continues in the direction of beacons BIB and AHA. The course of this example of simulation is captured in the training diagram via the following abbreviated formats of flight plans:

TABLE 1 ABBREVIATED FORMAT OF FLIGHT PLANS

\begin{tabular}{|c|c|c|c|c|}
\hline TA & NB 1 & NB 2 & NB 3 & NB 4 \\
& EFL 1 & EFL 2 & EFL 3 & EFL 4 \\
IAS & ETP 1 & ETP 2 & ETP 3 & ETP 4 \\
\hline JAS39 & CDE & BIB & AHA & ABC \\
& FL 80 & FL 80 & FL 100 & FL 100 \\
$405 \mathrm{kn}$ & $08: 05$ UTC & $08: 09$ UTC & $08: 11$ UTC & $08: 15$ UTC \\
\hline A319 & BCD & GIG & OHC & DEF \\
& FL 80 & FL 80 & FL 80 & FL 80 \\
$450 \mathrm{kn}$ & $08: 05$ UTC & $08: 09$ UTC & 08:10 UTC & 08:14 UTC \\
\hline
\end{tabular}

The displayed abbreviated flight plan formats present informational value about planed profiles of planes, displayed in Figure No. 1. The first column contains information about the type of aircraft (TA) and the planned indicated air speed (IAS). In the presented example, the simulation is participated in by a JAS 39 Gripen plan (the indicated air speed is $405 \mathrm{kn}$ ), and another of the planes in this case is an Airbus A-319 CJ (the indicated air speed is $450 \mathrm{kn}$ ). Each of the other columns provides three pieces of information related to passing the particular beacon: name of beacon (NB), estimated flight level (EFL) and the estimated time of passing (ETP).

For the task of automatic identification of conflict situations involving planes crossing on routes, we will use the specific characteristics of the polygon, particularly the space enclosed by four internal beacons - conflict zones. During a closer examination of the proposed polygon, we discover that the mutual distance between two planes, which are located in this zone at the same time, will reach values in the interval of $7 \mathrm{NM}$ to $0 \mathrm{NM}$ (Nautical miles). It can also be stated that if at a certain moment in time at least one of the planes will be located inside this zone, then the horizontal distance between another of the pair of planes will be greater than 5 NM.

Based on this finding, it is possible (in conditions of basic training) to resort to certain simplification and to declare that reduction of the horizontal distance between the two planes on these routes will occur at the moment when both planes are located inside the conflict zone. If they also fly at the same flight levels, then the vertical distance between them will be reduced, and a conflict situation will arise. Therefore, we are pointing out that conflict between planes on crossing routes can occur if there are two planes, which in the same moment are located in the conflict zone, and the reduction of the vertical distance between the the planes is also confirmed. Generally, the task of finding planned conflicts between planes on crossing routes can be divided into two sub-tasks:

- determination of the mutual position of the two planes in relation to the conflict zone,

- determination of the conflict nature of levels.

Each pair of planes located on crossing paths will be subjected to examination based on the sequence of the solutions for the aforementioned sub-tasks. The result of the examination is the finding that between planes on crossing routes in the particular simulation of an air situation, a conflict occurs, with specification of the circumstances of the conflict (by setting an interval in which the conflict occurs and identifying conflicting flight levels). Based on the analysis of conducting of training of military and civilian air traffic controllers, it was decided to resolve the task of optimisation of air traffic control training by using a knowledge approach through the proposed expert system [2]. This part of the work focuses on fulfilment of the base of knowledge of this system with rule systems [4], which identify the presence of conflict in selected simulations. Knowledge in rule systems is represented via rules, which can have the following general formats, for example:

\section{if (condition) then (conclusion) \\ if (situation) then (action) \\ if (condition) then (conclusion) and (action) \\ if (condition) then (consequencel) else \\ (consequence2)}

\section{SOLUTION IMPLEMENTATION}

Generally said, the process of conflict identification between planes A and B has been established with the help of following rule:

if $(I k \neq\{\} \wedge F k \neq\{\})$ then (yesCONF)

if $(I k=\{\} \vee F k=\{\})$ then $($ noCONF $)$, where:

Ik... interval of the horizontal limit reduction 
Fk... set of conflict flight levels achieved by planes within the interval of the horizontal limit reduction

$\checkmark$...or

$\wedge$...and also

yesCONF...conflict identified

noCONF... conflict not identified

The conflict identification between planes on crossing routes within the polygon is a special type of the task, when the interval Ik and the set of conflict flight levels Fk can be defined simply. The task has been divided into four sub-tasks:

- implementation of necessary data in the abbreviated format of flight plans,

- identification of crossing intervals of planes A and B (IkA, IkB) and a search for their penetration - Ik,

- the task of setting up sets of crossing flight levels of planes $\mathrm{A}$ and $\mathrm{B}$ (FkA and $\mathrm{FkB}$ ) and finding their penetration - Fk,

- task of examining the conflict situation between planes on crossing routes.

\section{A. Implementation of necessary data in the abbreviated format of flight plans}

Following essential implementation of necessary data (displaying of hidden levels (HIDFL) and clarifying time date on pass beacons), which was done with the help of a rule algorithm and calculation using models, which are used for the needs of creating simulation of planes (BADA models), the original abbreviated formats of flight plans receive this form:

TABLE 2 RECALCULATED FORMAT OF FLIGHT PLANS

\begin{tabular}{|l|l|l|l|l|}
\hline TA & NB 1 & NB 2 & NB 3 & NB 4 \\
& & HIDFL & & \\
IAS & EFL 1 & EFL 2 & EFL 3 & EFL 4 \\
& METP 1 & METP 2 & METP 3 & METP 4 \\
\hline JAS39 & CDE & BIB & AHA & ABC \\
& & FL 90 & & \\
& FL 80 & FL 80 & FL 100 & FL 100 \\
& $08: 05$ UTC & $08: 09$ UTC & 08:11 UTC & 08:15 UTC \\
& $08: 05: 00$ & $08: 09: 26$ & 08:11:03 & 08:15:32 \\
& UTC & UTC & UTC & UTC \\
\hline A319 & BCD & GIG & OHC & DEF \\
& FL 80 & FL 80 & FL 80 & FL 80 \\
$450 \mathrm{kn}$ & $08: 05$ UTC & 08:09 UTC & 08:10 UTC & 08:14 UTC \\
& $08: 05: 00$ & 08:09:00 & 08:10:20 & 08:14:20 \\
& UTC & UTC & UTC & UTC \\
\hline
\end{tabular}

In comparison with the abbreviated forms of flight plans displayed via Table No. 1, it is apparent that the information describing the conditions of planes on relevant beacons has been expanded to include details regarding modification of estimated time of passing (METP) and the hidden flight level (HIDFL).

The recalculated format of flight plans was realized by using Base of Aircraft Data models (BADA). These Aircraft Performance Models (APM) have been developed for use in aircraft trajectory simulations in the air traffic modelling through active cooperation with aircraft manufacturers and operating airlines. Information and data contained in BADA is designed with regard to uncertainty in measurement.

\section{B. Identification of crossing intervals of planes $A$ and B (IkA,}

$I k B)$ and a search for their penetration (Ik)

The crossing levels of planes A and B (IkA, IkB) represent entry and exit times of planes from the conflict zone. Due to greater demands for precise accuracy of these time details, it has been decided to use time details in the METP format (hh:mm:ss).

The crisis interval of plane A has been set as follows:

IkA = 〈METP 2(A; METP 3(A) >, where:

IkA...crisis interval of plane A

METP 2(A)... modification of estimated passing time of NB 2 by plane $A$

METP 3(A)... modification of estimated passing time of NB 3 by plane $\mathrm{A}$

The following applies similarly:

IkB = 〈 METP 2(B); METP 3(B) >, where:

IkB...crisis interval of plane B

METP 2(B)... modification of estimated passing time of NB 2 by plane $\mathrm{B}$

METP 3(B)... modification of estimated passing time of NB 3 by plane $\mathrm{B}$

Following achievement for the specific values of the examined simulation example, the following values of crisis intervals IkA and $\mathrm{IkB}$ were obtained:

\section{IkA $=\langle 08: 09: 26 ; 08: 11: 03>$ \\ IkB $=\langle 08: 09: 00 ; 08: 10: 20\rangle$}

Sought penetration of crisis intervals of planes IkA and IkB have been named the crisis interval of simulation - Ik and with the help of rule systems, the conditions they can acquire within simulation have been defined as follows:

1) Interval Ik is an empty set:

if $(\operatorname{METP} 3(A)<\operatorname{METP} 2(B))$ or

$(\operatorname{METP} 3(B)<(M E T P 2(A))$

then $(I k=\{\})$

2) Interval Ik is not an empty set:

if $($ METP $3(A) \geq \operatorname{METP} 2(B))$ or

$(\operatorname{METP} 3(B) \geq(\operatorname{METP} 2(A))$ then $(I k \neq\{\})$

and $(\mathrm{Ik}=\langle\operatorname{METP} 2(h) ; \operatorname{METP} 3(l)>)$, where: 
METP 2(A)... modification of estimated passing time of NB 2 by plane $A$

METP 2(B)... modification of estimated passing time of NB 2 by plane $\mathrm{B}$

METP 3(A)... modification of estimated passing time of NB 3 by plane $A$

METP 3(B)... modification of estimated passing time of NB 3 by plane $\mathrm{A}$

METP 2(h)...highest value of modified estimated passing time NB 2

METP 3(1)... lowest value of modified estimated passing time NB 2

METP 2(h) is expressed as follows:

if $(\operatorname{METP} 2(A)>\operatorname{METP} 2(B))$ then

$(\operatorname{METP} 2(h)=\operatorname{METP} 2(A))$

if $(M E T P 2(A) \leq M E T P 2(B))$ then

$(\operatorname{METP} 2(h)=\operatorname{METP} 2(B))$

METP 2(1) is expressed as follows:

if $(\operatorname{METP} 3(A)<\operatorname{METP} 3(B))$ then

$(\operatorname{METP} 3(l)=\operatorname{METP} 3(A))$

if $(M E T P 3(A) \geq \operatorname{METP} 3(B))$ then

$(\operatorname{METP} 3(l)=\operatorname{METP} 3(B))$

Application of the above rule systems led to the following conclusions regarding the examined example of simulation:

1) Ik $\neq\{\}$

2) Ik $=\langle 08: 09: 26$; 08:10:20 $\rangle$

C. The task of setting up sets of crossing flight levels of planes $A$ and $B$ (FkA and $F k B$ ) and finding their penetration - $F k$

The crisis flight levels of planes A and B have been named as the flight levels that the planes achieve within the particular situation when passing the conflict zone.

The set of crisis flight levels of plane A has been defined as follows:

FkA $=\{$ HIDFL(A) ; EFL 2(A); EFL 3(A) $\}$, where:

FkA... set of crisis flight levels of plane A

HIDFL(A)...hidden flight level of plane A

EFL 2 (A)...estimated flight level of plane A on position NB 2

EFL 3 (A)...estimated flight level of plane A on position NB 3

The following applies similarly:

FkB $=\{$ HIDFL(B) ; EFL 2(B); EFL 3(B) $\}$, where:

FkB... set of crisis flight levels of plane B

HIDFL(B)...hidden flight level of plane B

EFL 2(B)... estimated flight level of plane B on position NB

2

EFL 3(B)... estimated flight level of plane B on position NB 3
Following application on the examined example of simulation (achievement for a specific value), the following was arrived at:

FkA $=\{$ FL $90 ;$ FL $80 ;$ FL 100$\}$

FkB $=\{$ FL $80 ;$ FL 80$\}$

The penetration of sets of crisis flight levels of plans A and $\mathrm{B}$ has been identified by a set of crisis flight levels of simulation - Fk, and rule systems have been used to describe the conditions that the particular set could achieve:

1) Set Fk is empty:

if $(F k A \cap F k B=\{\})$ then $(F k=\{\})$

2) Set Fk is not empty:

if $(F k A \cap F k B \neq\{\})$ then $(F k \neq\{\})$ and $(F k=\{E F L 23(A B)\})$, where:

EFL 23(AB)... penetration of sets FkA and FkB (identical values of estimated flight levels of planes $A$ and $B$ in position NB 2 and in position NB 3)

The following conclusions were arrived at by using the aforementioned rule systems for examination of the presented example of simulation:

1) $\mathrm{Fk} \neq\{\}$

2) $\mathrm{Fk}=\{\mathrm{FL} 80\}$

D. Task of setting up a rule system for examination of a conflict situation

Based on the findings regarding the occurrence of conflict on crossing routes, specified in the theoretical part of the work, the following rule has been established for identification of the conflict situation between planes A and B on crossing routes:

if $(I k \neq\{\} \wedge F k \neq\{\})$ then (yesCONF) and

$($ Ik $=\langle\operatorname{METP} 2(h) ; \operatorname{METP} 3(l)>)$ and

$(F k=\{E F L 23(A B)\})$

if $(I k=\{\} \vee F k=\{\})$ then $($ noCONF $)$

Therefore, it is apparent from the previous examination that the following applies for the examined simulation example: $\mathrm{Ik} \neq$ \{\}$\vee \mathrm{Fk} \neq\{\}$, then states fulfilment of the first condition of the rule system and confirmation of the existence of a conflict within the examined simulation example (yesCONF).

The circumstances surrounding the arising of the conflict have been described with the help of crisis interval of simulation Ik and set of crisis flight levels Fk:

$\begin{array}{ll}\text { 1) } & \text { IK }=\langle 08: 09: 26 ; 08: 10: 20> \\ \text { 2) } & \text { Fk }=\{\text { FL 80 }\}\end{array}$

\section{CONCLUSION}

This article has presented the use of rule systems within the expert system, intended for identification of conflicts between planes in selected types of simulation for the needs of basic training.

During the realisation of the solution, abbreviated formats of flight plans were presented, which were appropriately expanded 
to include necessary data. The other parts focused on identification of conditions in which conflict situations occur between two planes. For this purpose, rule systems were proposed for identification of the conditions in which reduction of the set minimum of horizontal or vertical distance away occurred.

In the final phase, a rule system was set up, which with final validity identifies the conflict between two planes on crossing paths, and if a conflict is identified, generates the circumstances of occurrence of this conflict.

The theoretical conclusions were presented in a specific example of simulation of planes on crossing paths, and it was discovered that a conflict would arise between these planes.

The scope of the task of automatic identification of conflict between planes in the selected basic types of simulation exceeds the capabilities of this article. In other parts of this task, it would be appropriate to expand the options of automatic identification of conflicts between planes to other types of simulations, when planes based on their positions in relation to each other encounter each other on the same or opposite-direction routes.

\section{REFERENCES}

[1] BARTOŠOVÁ, N. Temporary condition of military area air traffic control service. In: Proceedings of AIM 2009. Brno, 2009. ISBN 978-807231-696-0

[2] BARTOŠOVÁ, N. Optimisation method for air traffic controllers training. In: ICMT 2013 Proceedings, pp. (1227 - 1233). Brno, 2013. ISBN: 978-80-7231-917-6

[3] DOC 4444/L 4444 - Postupy pro letové navigační služby: Flight regulation. Praha: MD ČR, 2011. Č. j.:439/2011-220-SP/1

[4] DVOŘÁK, J. Rules on expert systems. [online]. Brno: VUT, 2014. [2014-09-18]. Accessible on:

www.uai.fme.vutbr.cz/ jdvorak/vyuka/es/RuleES.ppt 